\title{
Green's function approach to the lifetimes of image potential resonances at metal surfaces
}

\author{
S. S. Tsirkin, ${ }^{1,2, *}$ A. G. Borisov, ${ }^{1,3}$ and E. V. Chulkov ${ }^{1,2,4,5}$ \\ ${ }^{1}$ Donostia International Physics Center (DIPC), 20018 San Sebastián/Donostia, Basque Country, Spain \\ ${ }^{2}$ Tomsk State University, 634050 Tomsk, Russia \\ ${ }^{3}$ Institut des Sciences Moléculaires d'Orsay, ISMO, UMR 8214 CNRS-Université, Paris-Sud, Bâtiment 351, \\ Université Paris-Sud, 91405 Orsay CEDEX, France \\ ${ }^{4}$ Departamento de Física de Materiales UPV/EHU, Facultad de Ciencias Químicas, UPV/EHU, Apdo. 1072, \\ 20080 San Sebastián/Donostia, Basque Country, Spain \\ ${ }^{5}$ Centro de Física de Materiales CFM-MPC, Centro Mixto CSIC-UPVIEHU, 20080 San Sebastián/Donostia, Basque Country, Spain
}

(Received 5 April 2013; revised manuscript received 12 July 2013; published 31 July 2013)

\begin{abstract}
We present a theoretical study of the image potential resonances (IPRs) at metal surfaces. We develop the Green's functions approach allowing us to calculate binding energies $E_{n}$ and lifetimes $\tau_{n}$ of IPRs with high quantum numbers $n$ (up to 10 in this work). A systematic study is performed at the $\bar{\Gamma}$ point for the close-packed metal surfaces: $\mathrm{Cu}(111), \mathrm{Ag}(111), \mathrm{Au}(111), \mathrm{Al}(001), \mathrm{Al}(111), \mathrm{Be}(0001), \mathrm{Mg}(0001), \mathrm{Na}(110), \mathrm{Li}(110)$, and also at the $\overline{\mathrm{Y}}$ point on $\mathrm{Cu}(110)$. The calculated lifetimes of IPRs on close-packed surfaces demonstrate the scaling law $\tau_{n} \propto n^{3}$. Our results are in agreement with available experimental data. We show that at the $\overline{\mathrm{Y}}$ point on $\mathrm{Cu}(110)$ each quantum number $n$ corresponds to a pair of IPRs $n^{+}$and $n^{-}$, where the energy difference $E_{n+}-E_{n-}$ is proportional to $n^{-3}$. The lifetimes $\tau_{n+}$ and $\tau_{n-}$ differ significantly, however, they both obey the scaling law $\tau_{n \pm} \propto n^{3}$. Since the electrons trapped in the long-lived IPRs are strongly localized on the vacuum side, we argue that the inelastic electron-electron and electron-phonon scattering have a small contribution to the decay rate of these IPRs. The latter is dominated by the resonant electron transfer into the bulk.
\end{abstract}

DOI: 10.1103/PhysRevB.88.035449

PACS number(s): 73.20.-r, 72.10.-d

\section{INTRODUCTION}

Information about the lifetimes of electronic excitations is important for the description of many physical and chemical phenomena, such as charge and energy transport, ${ }^{1}$ interaction of atoms and molecules with surfaces, ${ }^{2}$ and catalytic reactions. ${ }^{3}$ At metal surfaces, the electrons can be excited into the intrinsic surface states, ${ }^{4,5}$ image potential states (IPSs), ${ }^{6-14}$ quantum well states, ${ }^{15-19}$ as well as in adsorbate-induced states. ${ }^{20-26}$ Surface states originate from the crystal symmetry breaking near the surface and are localized in a few outermost atomic layers. IPSs are formed at metal surfaces by the image potential: 27,28 an attractive interaction between the electron and polarization charge it induces at the surface. IPSs are mostly localized on the vacuum side in front of the surface. At the $\bar{\Gamma}$ point of the surface Brillouin zone (SBZ), the energies $E_{n}$ of the IPSs form a Rydberg-type series, converging to the vacuum level $E_{\text {vac }}$ (Refs. 29 and 30):

$$
E_{n}=E_{\mathrm{vac}}-\frac{0.85 \mathrm{eV}}{(n+\delta)^{2}},
$$

where $n=1,2, \ldots$ is the principal quantum number and $\delta$ the quantum defect. Due to their well-known spectrum, IPSs have been widely used as a model system for both theoretical and experimental investigations of the dynamics of electronic excitations at metal surfaces. ${ }^{6-14,31}$

When surface states or IPSs are degenerate with bulk bands, the electrons localized in these states can escape via energy-conserving transitions into the substrate. The quasistationary surface state resonances or image potential resonances (IPRs) are then formed. The total decay rate $\Gamma=\hbar / \tau$ ( $\tau$ being the lifetime) of an electronic excitation in the quasistationary IPR state is thus determined by four contributions: ${ }^{26,31}$ inelastic electron-electron scattering ${ }^{4,5}$ (decay rate $\Gamma_{e e}$ ), electron-phonon interaction ${ }^{32,33}\left(\Gamma_{e p}\right)$, electron-defect scattering ${ }^{34}\left(\Gamma_{e d}\right)$, and energy-conserving resonant one-electron tunneling into the bulk ${ }^{35-38}\left(\Gamma_{1 e}\right)$. The one-electron transfer into the substrate is thought to be faster than the many-body decay and so to dominate the population decay of the quasistationary states at low defect surfaces. ${ }^{35,39,40}$

Early experimental observations concerned the IPRs with low quantum numbers $(n \leqslant 3)$, ${ }^{6,8,30,36,41}$ Indeed, the IPRs with high quantum numbers have dense energy spectrum and high-energy resolution is then necessary to distinguish these states as separate photoemission peaks. It is only recently that advances in the time-resolved two-photon photoemission (TR2PPE) allowed us to study energies and lifetimes of IPRs with high $n .^{39,40}$ Theoretical studies of the lifetimes of IPRs reported so far addressed resonant decay into the bulk treated with the one-electron wave-packet propagation (WPP) technique. ${ }^{35-38}$ Good agreement with the experimental data ${ }^{6,8,30,36,37,42}$ has been obtained, allowing detailed discussion of the role of the resonant one-electron tunneling into the substrate.

As any time-domain approach, the WPP becomes computationally extremely heavy for the high- $n$ IPRs where the mandatory high-energy resolution translates to extremely long propagation times. Moreover, the high- $n$ IPRs have the wave functions strongly extending into the vacuum side with an average electron-surface distance growing as $n^{2}$. The large spatial mesh is then required, further hindering application of the WPP. That is why we develop here an alternative approach allowing an efficient study of the lifetimes of IPRs on metallic surfaces. It is based on the the Greens's functions (GF) technique, as applied by Jurczyszyn to determine the energies of IPRs on metal surfaces; ${ }^{43-45}$ however, the lifetimes of the IPRs were not addressed in these works. We show that the GF method outperforms the WPP in that it allows us to study the high- $n$ IPRs, and it offers higher numerical stability in 
determining the lifetimes of the IPRs over the entire $n$ series. Additional advantage of the GF technique is that, different from WPP, it is not restricted to one-electron transitions, but the many-body decay channels due to electron-phonon and electron-electron scattering can be included into the treatment through the corresponding corrections into the GF. However, at the present stage we have not attempted to evaluate the many-body contribution, leaving it for future work.

We report the binding energies and lifetimes of IPRs at the $\bar{\Gamma}$ point on a number of close-packed surfaces: $\mathrm{Cu}(111)$, $\mathrm{Ag}(111), \mathrm{Au}(111), \mathrm{Al}(001), \mathrm{Al}(111), \mathrm{Be}(0001), \mathrm{Mg}(0001)$, $\mathrm{Na}(110), \mathrm{Li}(110)$. When available, we retrieve results of the WPP calculations within the computational accuracy. Our results are also in agreement with experimental data, including recent experiments for high- $n$ IPRs on $\mathrm{Al}(001) .{ }^{39}$ Further, we apply our method to study the energies and lifetimes of IPRs at the $\overline{\mathrm{Y}}$ point on $\mathrm{Cu}(110)$. Indeed, most of the reported experimental ${ }^{6,8,11,30,36,37,39-42,46-50}$ and theoretical $^{35,37,38,43-45,48-51}$ studies of energies and lifetimes of image potential states and resonances at metal surfaces are restricted to the vicinity of the $\bar{\Gamma}$ point of the surface Brillouin zone. The energies of IPSs near the surface Brillouin zone boundary were measured only in a few works. ${ }^{52-55}$ Theoretically, the energy dispersion of IPSs on the (110) surfaces of fcc metals along the $\overline{\Gamma Y}$ direction has been analyzed with a multiple-reflection model. ${ }^{56,57}$ The lifetimes of IPRs at the $\bar{Y}$ point of (110) surfaces of fcc metals have not been reported so far.

We perform our calculations in the geometry of a semiinfinite crystal, which correctly incorporates the continuum of bulk electronic states, in contrast to the slab geometry, where the bulk states are discrete. The electron interaction with close-packed surfaces is described by a pseudopotential which is a function of only the electron coordinate in the direction perpendicular to the surface. For $\mathrm{Cu}(111), \mathrm{Ag}(111), \mathrm{Au}(111)$, $\mathrm{Be}(0001)$ and $\mathrm{Na}(110), \mathrm{Li}(110)$, we use the potential, introduced in Refs. 51 and 58, and validated in numerous calculations of the lifetimes of surface states, ${ }^{4,5,26,59,60}$ image potential states, ${ }^{4,26,33,48,50,61}$ and IPRs. ${ }^{35,36,50}$ In the case of jelliumlike surfaces of $\mathrm{Al}(001), \mathrm{Al}(111)$, and $\mathrm{Mg}(0001)$, we find that this potential should be improved for more accurate description of their electronic structure. Thus, we modify the form of the potential for these surfaces. The $\mathrm{Cu}(110)$ surface is described by the pseudopotential, ${ }^{62,63}$ varying in the directions [110] (perpendicular to the surface) and [001] (parallel to atomic rows on the surface).

The pseudopotentials used in the calculations are described in Sec. II A. The construction of the GF is presented in Sec. II B. The technique of extracting the binding energies and lifetimes of IPRs is given in Sec. II C. Section III is devoted to the results and their discussion. The main conclusions are summarized in Sec. IV. Unless otherwise stated, atomic units (a.u.) are used in the paper, i.e., $e^{2}=\hbar=m_{e}=1$, and the energies are measured relative to the vacuum level $E_{\mathrm{vac}}$.

\section{METHOD}

\section{A. Pseudopotential}

In what follows, we use the coordinate system with the $z$ axis perpendicular to the surface $(x, y)$ plane, $z=0$ corresponds to the position of the outermost atomic layer, and positive $z$ corresponds to an electron being in vacuum. The electronic structure of close-packed surfaces is described with a model pseudopotential, the only function of the $z$ coordinate. The potential inside the crystal $(z<0)$ is periodic and has the form

$$
V(z)=V_{0}+V_{g} \cos \left(\frac{2 \pi}{a_{s}} z\right),
$$

where $a_{s}$ is the interlayer spacing, $V_{g}$ and $V_{0}$ are Fourier components of the bulk potential, determining the width and the position of the energy gap at the $\bar{\Gamma}$ point. The bulk potential given by Eq. (2) smoothly joins the vacuum potential for $z>0$. Far from the surface, the latter converges to the image potential

$$
V(z)=-\frac{1}{4\left(z-z_{\text {im }}\right)},
$$

where $z_{i m}$ is the position of the image plane

For $\mathrm{Ag}(111), \mathrm{Cu}(111), \mathrm{Au}(111), \mathrm{Be}(0001), \mathrm{Na}(110)$, and $\mathrm{Li}(110)$, we use the vacuum part of the potential as proposed in Refs. 51 and 58:

$$
\begin{aligned}
& V(z)=A_{20}+A_{2} \cos (\beta z), \quad 0<z<z_{1} \\
& V(z)=A_{3} \exp \left[-\alpha\left(z-z_{1}\right)\right], \quad z_{1}<z<z_{i m} \\
& V(z)=\frac{\exp \left[-\lambda\left(z-z_{i m}\right)\right]-1}{4\left(z-z_{i m}\right)}, \quad z_{i m}<z
\end{aligned}
$$

where $z_{1}=5 \pi / 4 \beta$. Parameters of the potential are set such that it allows us to reproduce the essential features of the projected band structure: energy position of the projected band gap, and energies of the surface state, and of the $n=1$ IPS ( $n=1$ IPR). For $\mathrm{Cu}(111), \mathrm{Au}(111), \mathrm{Be}(0001), \mathrm{Na}(110)$, and $\operatorname{Li}(110)$, we use the parametrization reported in Refs. 51 and 58. For $\operatorname{Ag}(111)$, a new set of parameters was defined $\left(V_{0}=-9.482 \mathrm{eV}, V_{g}=4.1582 \mathrm{eV}, A_{2}=4.100, \beta=2.5606\right)$ allowing accurate description of recent experimental data. ${ }^{39,48}$

The above form of the pseudopotential has been widely used for the description of the band structure of close-packed metal surfaces. $4,5,26,33,35,36,48,50,59-61$ However, we find that this potential is not very accurate for the description of the band structure of $\mathrm{Al}(001), \mathrm{Al}(111)$, and $\mathrm{Mg}(0001)$. Therefore, we modify the form of the potential given by Eq. (4). For $0<z<z_{1}$ we use

$$
V(z)=A_{20}+A_{2} \cos \left(\beta z+(\beta z)^{3}\right) .
$$

Here, the $z_{1}$ point is determined by $\beta z_{1}+\left(\beta z_{1}\right)^{3}=\frac{5 \pi}{4}$. With such a potential, we reproduce the surface state and the $n=2$ IPR correctly. We use the $n=2$ IPR for the fitting of the potential because the $n=1$ IPR is too broad on these surfaces, and its position can not be accurately determined. The corresponding parameters for $\mathrm{Al}(001)$ are $\beta=1.0834$ bohrs $^{-1}$, $A_{2}=2.268 \mathrm{eV}$. The other parameters are obtained from the requirement of the continuity of the potential and its first derivative. For $\mathrm{Al}(111)$ and $\mathrm{Mg}(0001)$ surfaces, no experimental data are available on IPRs. Early first-principles slab-model-based calculations $^{51,58}$ of the binding energies of the resonances could not give accurate values because of the insufficient slab thickness used (19-27 layers). We then rely on the following:

(i) Similarity of the electronic structure of $\mathrm{Al}(111)$ and $\mathrm{Mg}(0001)$ with that of $\mathrm{Al}(001)$ where in all cases the width of 
the bulk gap $V_{g}$ is very small, and the upper gap edge $E_{\text {up }}$ is well below the vacuum level;

(ii) Recent experimental data ${ }^{40}$ showing that Eq. (1) is valid for IPRs with numbers $n \geqslant 2$ for $\mathrm{Al}(100)$ with the quantum defect $\delta=0$.

To find a parametrization of the potential for $\mathrm{Al}(111)$ and $\operatorname{Mg}(0001)$ surfaces, we thus suppose that the series of IPRs at these surfaces is also described by Eq. (1) with $\delta=0$. With this assumption, we find the parameters, which reproduce the $n=2$ IPR energy $E_{2}=-212.6 \mathrm{meV}$, along with the surface state: $\beta=1.170$ bohrs $^{-1}, A_{2}=2.100 \mathrm{eV}$ for $\mathrm{Al}(111)$; and $\beta=0.9189$ bohrs $^{-1}, A_{2}=1.260 \mathrm{eV}$ for $\mathrm{Mg}(0001)$.

For the $\mathrm{Cu}(110)$, we set the $x$ and $y$ axes along the [110] and [001] directions, respectively, and we use the two-dimensional pseudopotential, ${ }^{62,63}$ varying in the [110] $(z)$ and [001] $(y)$ directions. This pseudopotential has been already validated with calculations of many-body lifetimes of electronic excitations in surface states ${ }^{64,65}$ and IPSs (Ref. 65) at the $\bar{Y}$ point on the (110) surfaces of fcc metals. Inside the crystal $(z<0)$, the potential is periodic and has the form

$$
V(y, z)=V_{0}+2 V_{g} \cos \left(\frac{2 \sqrt{2} \pi}{a} z\right) \cos \left(\frac{2 \pi}{a} y\right),
$$

where $a$ is the lattice parameter, $V_{g}$ and $V_{0}$ are Fourier components of the bulk potential, determining the width and the position of the energy gap at the $\bar{Y}$ point. For $z>0$ the pseudopotential smoothly joins the vacuum part given by

$$
V(y, z)=V^{(0)}(z)+V^{(1)}(z) \cos \left(\frac{2 \pi}{a} y\right) .
$$

Far from the surface, the potential $V^{(1)}(z)$ decays exponentially while the $V^{(0)}(z)$ converges to the image potential given by Eq. (3).

\section{B. Green's function matching method}

In this section, we detail the calculation of the GF of the semi-infinite crystal using the Green's function matching method $^{66}$ allowing us to construct the GF of the whole system from the GFs, defined only in its distinct parts. We set down the equations for the case of close-packed surfaces, where the electronic structure is described by a one-dimensional pseudopotential. For $\mathrm{Cu}(110)$, the GF is calculated in an analogous way. However, due to the $y$ dependence of the pseudopotential, the formalism becomes cumbersome, and we do not feel it necessary to detail the corresponding equations here.

With the Green's function matching method, the system is divided into three parts: the bulk region $(z \leqslant 0)$, the image potential region $\left(0 \leqslant z \leqslant L_{\mathrm{IP}}\right)$, and the vacuum region $\left(L_{\mathrm{IP}} \leqslant z\right)$. The value $L_{\mathrm{IP}}$ is chosen sufficiently large ( $\sim 1500$ a.u.), so that the local density of states $\operatorname{LDOS}(\omega, z)=$ $-\operatorname{Im}[G(z, z, \omega)] / \pi$ decays at $z \rightarrow L_{\mathrm{IP}}$ for the energy $\omega$ corresponding to the highest studied IPR with $n=10$. For the above three regions, we calculate the corresponding GFs $G_{b}\left(z \leqslant 0, z^{\prime} \leqslant 0, \omega\right), G_{i}\left(0 \leqslant z \leqslant L_{\mathrm{IP}}, 0 \leqslant z^{\prime} \leqslant L_{\mathrm{IP}}, \omega\right)$, and $G_{v}\left(L_{\mathrm{IP}} \leqslant z, L_{\mathrm{IP}} \leqslant z^{\prime}, \omega\right)$, satisfying the Neumann boundary conditions at $z=0$ and $z=L_{\mathrm{IP}}$ :

$$
\left.\partial_{z} G_{b, i, v}\left(z, z^{\prime}, \omega\right)\right|_{z=0, z=L_{\mathrm{IP}}}=0 .
$$

Provided $G_{b}, G_{i}$, and $G_{v}$, the GF of the entire system can be constructed following the procedure of Ref. 66. In the one-dimensional case we have, for $0<z, z^{\prime}<L_{\mathrm{IP}}$,

$$
\begin{aligned}
G\left(z, z^{\prime}\right)= & G_{i}\left(z, z^{\prime}\right)-\left\{G_{i}(z, 0) K_{11} G_{i}\left(0, z^{\prime}\right)+G_{i}\left(z, L_{\mathrm{IP}}\right) K_{22}\right. \\
& \left.\times G_{i}\left(L_{\mathrm{IP}}, z^{\prime}\right)-2 G_{i}(z, 0) K_{12} G_{i}\left(L_{\mathrm{IP}}, z^{\prime}\right)\right\}
\end{aligned}
$$

and, for $z, z^{\prime}<0$,

$$
G\left(z, z^{\prime}\right)=G_{b}\left(z, z^{\prime}\right)-G_{b}(z, 0) K_{11} G_{b}\left(0, z^{\prime}\right) .
$$

Here,

$$
\begin{aligned}
K_{11}= & {\left[G_{i}\left(L_{\mathrm{IP}}, L_{\mathrm{IP}}\right)+G_{v}\left(L_{\mathrm{IP}}, L_{\mathrm{IP}}\right)\right] / M, } \\
K_{22}= & {\left[G_{i}(0,0, \omega)+G_{b}(0,0)\right] / M, } \\
K_{12}= & K_{21}=G_{i}\left(0, L_{\mathrm{IP}}\right) / M, \\
M= & {\left[G_{i}\left(L_{\mathrm{IP}}, L_{\mathrm{IP}}\right)+G_{v}\left(L_{\mathrm{IP}}, L_{\mathrm{IP}}\right)\right]\left[G_{i}(0,0, \omega)\right.} \\
& \left.+G_{b}(0,0)\right]-G_{i}^{2}\left(0, L_{\mathrm{IP}}\right) .
\end{aligned}
$$

The argument $\omega$ was omitted for brevity in Eqs. (11)-(16), implying that all Green's functions are calculated at the same frequency.

To obtain $G_{b}\left(z, z^{\prime} ; \omega\right)$, first, we calculate the eigenvalues $\epsilon_{n, k_{z}}$ and eigenfunctions

$$
\psi_{n, k_{z}}(z)=\frac{1}{\sqrt{L}} \sum_{g_{z}} C_{n, k_{z}}^{g_{z}} e^{i\left(k_{z}+g_{z}\right) z}
$$

of the bulk one-dimensional (1D) crystal, satisfying the Schrödinger equation with the potential (2). Here, $L$ is the normalization length, $g_{z}=\frac{2 \pi}{a_{s}} m$ (m an integer), and $\sum_{g_{z}}\left|C_{n, k_{z}}^{g_{z}}\right|^{2}=1$. Then, we construct the bulk GF

$$
\begin{aligned}
G_{b}\left(z, z^{\prime}, \omega\right)= & \frac{2}{\pi} \sum_{n} \int_{0}^{\pi / a} \sum_{g_{z}} C_{n, k_{z}}^{g_{z}} \cos \left[\left(k_{z}+g_{z}\right) z\right] \\
& \times \sum_{g_{z}^{\prime}} C_{n, k_{z}}^{g_{z}^{\prime}} \cos \left[\left(k_{z}+g_{z}^{\prime}\right) z^{\prime}\right] \frac{d k_{z}}{\omega-\epsilon_{n, k_{z}}} .
\end{aligned}
$$

The Green's function for $L_{\mathrm{IP}}>z, z^{\prime}>0$ is determined by

$$
G_{i}\left(z, z^{\prime}, \omega\right)=\sum \frac{\phi_{m}(z) \phi_{m}\left(z^{\prime}\right)}{\omega-\varepsilon_{m}},
$$

where the wave functions $\phi_{m}(z)$ and energies $\varepsilon_{m}$ are obtained by diagonalizing the Hamiltonian

$$
H_{l l^{\prime}}=\int_{0}^{L_{\mathrm{IP}}} f_{l}(z)\left(-\frac{\partial_{z}^{2}}{2}+V(z)\right) f_{l^{\prime}}(z) d z
$$

in the basis set $f_{0}=\sqrt{1 / L_{\mathrm{IP}}}, f_{l}=\sqrt{2 / L_{\mathrm{IP}}} \cos \left(\pi l z / L_{\mathrm{IP}}\right)$, $l \geqslant 1$.

Finally, for $z>L_{\mathrm{IP}}$ the image potential is assumed to converge to the vacuum level, and hence the Green's function satisfying (10) is

$$
G_{v}\left(L_{\mathrm{IP}}, L_{\mathrm{IP}}, \omega\right)=-\frac{2 i}{\sqrt{2 \omega}},
$$

where we choose the square root with the positive imaginary part. In order to avoid singularities, we calculate the Green's function at complex frequencies $\omega$ in the vicinity of the 
real axis, where the imaginary part $\operatorname{Im}[\omega]=0.1 \mathrm{meV}$ is considerably smaller than the widths of the studied resonances.

\section{Determination of binding energies and lifetimes}

We shall analyze our results in terms of the "image potential" GF, defined as

$$
G_{\mathrm{IP}}(\omega)=\int_{z_{i m}}^{L_{\mathrm{IP}}} G(z, z, \omega) d z+i \frac{L_{\mathrm{IP}}}{\sqrt{2 \omega}},
$$

where the imaginary part of $G_{\mathrm{IP}}(\omega<0)$ gives the energyresolved probability to find an electron above the metal surface. The second term is included in (22) to cancel the nonresonant contribution of the free-electron states. We would also use below the quantity dubbed as the "surface" GF:

$$
G_{S}^{\lambda}(\omega)=\int_{-\infty}^{z_{i m}} e^{\left(z-z_{i m}\right) / \lambda} G(z, z, \omega) d z
$$

with penetration depth $\lambda$ set to be of the order of several atomic layers.

The quasistationary states (here the IPRs) are associated with the poles of the GF, so that the extraction of their energies and lifetimes is based on the fit of the $G_{\mathrm{IP}}(\omega)$ with an analytical expression explicitly including the resonant and nonresonant contributions.

$$
\tilde{G}_{\mathrm{IP}}(\omega)=\frac{1}{\omega-\omega_{n}}+\sum_{l=0}^{2} A_{l}\left(\omega-\omega_{n}\right)^{l} .
$$

Here, $\omega_{n}$ and $A_{l}$ are the complex fitting parameters. The first term in (24) corresponds to the contribution of the pole at complex frequency $\omega_{n}$, and second term describes the slowly varying nonresonant part. The energies of the IPRs are then given by $E_{n}=\operatorname{Re}\left[\omega_{n}\right]$ and the corresponding decay rates are given by $\Gamma_{n}=-2 \operatorname{Im}\left[\omega_{n}\right]$, so that $\tau_{n}=1 / \Gamma_{n}=-1 / 2 \operatorname{Im}\left[\omega_{n}\right]$ are the lifetimes of the IPRs. In practice, the fitting procedure is applied independently in the vicinity of each $n$th IPR. It is worth noting that the normalization of the pole contribution as $1 /\left(\omega-\omega_{n}\right)$ implies that the corresponding decay rate $\Gamma_{n}$ is very small. The pole in the GF is close to the real axis and the resonance wave function is very much that of the bound state ( $n$th IPS) residing mainly on the vacuum side. This assumption is fully justified by the results below, and it appears extremely handy in reaching stable numerical convergence of the fit given by Eq. (24).

Note that for $\mathrm{Cu}(110)$, the GF depends on $z$ and $y$ and hence $G_{\text {IP }}$ is defined by

$$
\begin{aligned}
G_{\mathrm{IP}}(\omega)= & \int_{0}^{a} \frac{d y}{a} \int_{z_{i m}}^{L_{\mathrm{IP}}} G\left(y, z, y, z ; k_{\overline{\mathrm{Y}}}, \omega\right) d z \\
& +\sum_{g} \frac{i L_{\mathrm{IP}}}{\sqrt{2 \omega-\left(k_{\overline{\mathrm{Y}}}+g\right)^{2}}},
\end{aligned}
$$

where $g=2 \pi n / a, a$ is the lattice parameter, $n$ an integer, and $k_{\overline{\mathrm{Y}}}=\pi / a$.

\section{RESULTS AND DISCUSSION}

\section{A. Close-packed surfaces}

The (111) surfaces of silver and copper have similar band structure: the $n=1$ IPS is located in the bulk $L$ gap, while the
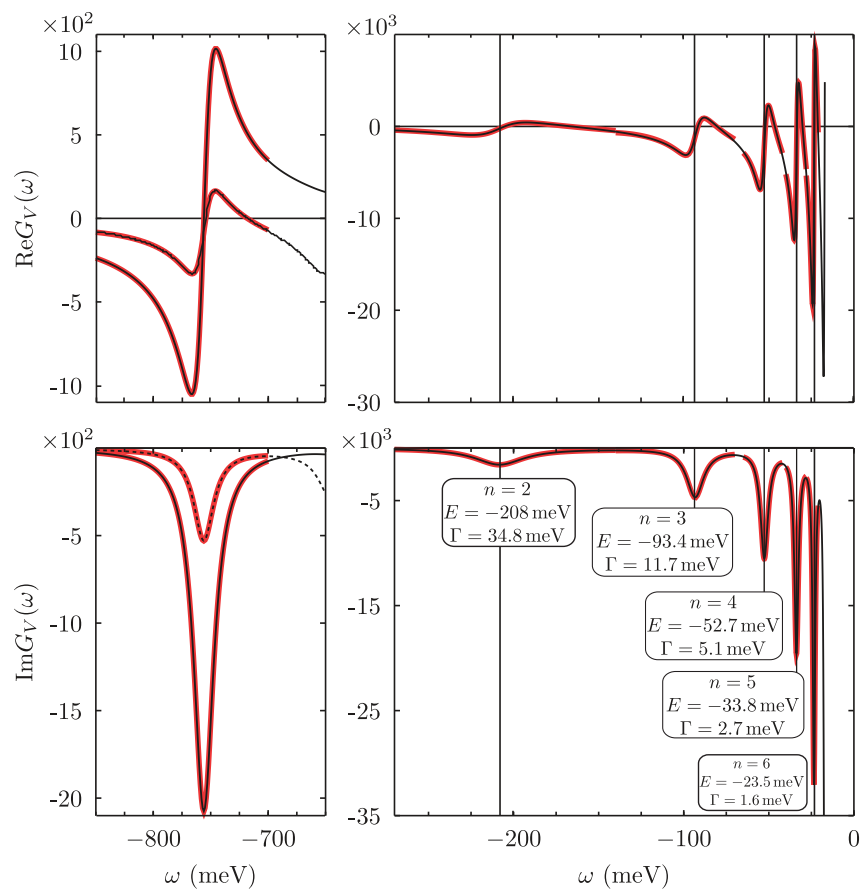

FIG. 1. (Color online) The real (upper panel) and imaginary (lower panel) parts of $G_{\mathrm{IP}}(\omega)$ for $\operatorname{Ag}(111)$. Black thin lines: the calculated dependence; thick red lines: the fits by function (24) in the vicinities of the resonances. Dashed lines in (a) represent $G_{S}(\omega)$.

higher IPSs with $n \geqslant 2$ lie in the projection of the bulk bands on the SBZ, so that the IPRs are formed at these energies.

The calculated Green's function of $\operatorname{Ag}(111)$ is presented in Fig. 1. As far as we do not consider inelastic scattering in the present calculations, the $n=1$ image potential state is represented by a pole on the real axis at $\omega_{n=1}=-756 \mathrm{meV}$. In the data shown in the left panels of Fig. 1, we have introduced a finite broadening $\Gamma_{n=1}=21 \mathrm{meV}$ by calculating the GF at $\operatorname{Im}[\omega]=\Gamma_{n=1} / 2$, which corresponds to the decay rate measured in the time-resolved two-photon photoemission. ${ }^{39}$ In the vicinity of the energy of the $n=1$ IPS, the image potential GF may be fitted with

$$
\begin{aligned}
& \tilde{G}_{S}^{\lambda}(\omega)=\sum_{l=0}^{2} A_{l}\left(\omega-\omega_{n=1}\right)^{l}+\frac{P_{S}}{\omega-\omega_{n=1}}, \\
& \tilde{G}_{\mathrm{IP}}(\omega)=\sum_{l=0}^{2} A_{l}\left(\omega-\omega_{n=1}\right)^{l}+\frac{P_{\mathrm{IP}}}{\omega-\omega_{n=1}},
\end{aligned}
$$

where $\omega_{n}, A_{l}, P_{S}$, and $P_{\mathrm{IP}}$ are the fitting parameters $\left(\omega_{n}, A_{l}\right.$ are complex and $P_{S}, P_{\mathrm{IP}}$ are real). We have obtained $P_{S}=0.20$ and $P_{\mathrm{IP}}=0.80$ which define the weights of the $n=1$ IPS in the surface $\left(z<z_{i m}\right)$ and in the image potential $\left(z>z_{\text {im }}\right)$ regions correspondingly. These values are in accordance with findings of Ref. 48, where the weights were calculated by integrating the squared wave function of the $n=1$ IPS. The $n \geqslant 2$ image potential resonances correspond to the poles of $G_{\mathrm{IP}}(\omega)$ at complex energies $\omega_{n}=E_{n}-i \Gamma_{n} / 2$. In Fig. 1, one can see that in the vicinities of the resonances, $G_{\mathrm{IP}}(\omega)$ can be accurately approximated by Eq. (24), where the residue of the pole equals unity. 
TABLE I. The energies $E_{n}(\mathrm{meV})$ and lifetimes $\tau_{n}$ (fs) of IPRs. Present results are compared with wave-packet propagation (WPP) calculations and with experimental data. $E_{\text {up }}$ is the position of the upper edge of the projected band gap, $V_{g}$ is the width of the projected band gap.

\begin{tabular}{|c|c|c|c|c|c|}
\hline \multirow[b]{3}{*}{$n$} & \multicolumn{5}{|c|}{$\begin{array}{c}\operatorname{Ag}(111) \\
E_{\text {up }}=-0.65 \mathrm{eV}, V_{g}=4.26 \mathrm{eV}\end{array}$} \\
\hline & \multicolumn{2}{|c|}{ This study } & \multirow{2}{*}{$\begin{array}{c}\text { WPP (Ref. 35) } \\
\tau_{n}\end{array}$} & \multicolumn{2}{|c|}{ Experiment } \\
\hline & $E_{n}$ & $\tau_{n}$ & & $E_{n}$ & $\tau_{n}$ \\
\hline 1 & -756 & & & $-756 \pm 3$ (Ref. 39) & $31.0 \pm 1.5($ Ref. 39$), 32($ Ref. 42$)$ \\
\hline 2 & -208 & 18.9 & 17.3 & $-212 \pm 10($ Ref. 39) & $23 \pm 2($ Ref. 39$), \leqslant 20($ Ref. 42$)$ \\
\hline 3 & -93.4 & 56.2 & 51.8 & $-91 \pm 2($ Ref. 39) & $65 \pm 5$ (Ref. 39), 65 (Ref. 42) \\
\hline 4 & -52.7 & 128 & 122 & $-51 \pm 1($ Ref. 39) & $166 \pm 20($ Ref. 39) \\
\hline 5 & -33.8 & 246 & 246 & $-33 \pm 1$ (Ref. 39) & $393 \pm 40$ (Ref. 39) \\
\hline 6 & -23.5 & 419 & 396 & $-23 \pm 1$ (Ref. 39) & $683 \pm 80$ (Ref. 39) \\
\hline 7 & -17.3 & 661 & & $-17 \pm 1$ (Ref. 39) & $1088 \pm 140($ Ref. 39$)$ \\
\hline 8 & -13.2 & 978 & & & \\
\hline 9 & -10.5 & 1413 & & & \\
\hline 10 & -8.48 & 1915 & & & \\
\hline \multirow[t]{4}{*}{$\tau_{1}^{*}$} & & 1.94 & & & \\
\hline & \multirow{2}{*}{\multicolumn{5}{|c|}{$\begin{array}{c}\mathrm{Cu}(111) \\
E_{\text {up }}=-0.69 \mathrm{eV}, V_{g}=5.14 \mathrm{eV}\end{array}$}} \\
\hline & & & & & \\
\hline & \multicolumn{2}{|c|}{ This study } & WPP (Ref. 35) & \multicolumn{2}{|c|}{ Experiment } \\
\hline$n$ & $E_{n}$ & $\tau_{n}$ & $\tau_{n}$ & $E_{n}$ & $\tau_{n}$ \\
\hline 1 & -817 & & & $-820 \pm 50$ (Ref. 6$)$ & $18 \pm 5$ (Refs. 6 and 8 ) \\
\hline 2 & -214 & 17.0 & 16.2 & $-250 \pm 70($ Ref. 6$)$ & $14 \pm 3$ (Ref. 8), $17 \pm 5$ (Ref. 6) \\
\hline 3 & -94.9 & 52.1 & 49.1 & -95 (Ref. 8) & $40 \pm 6($ Ref. 8$)$ \\
\hline 4 & -53.4 & 121 & 118 & & \\
\hline 5 & -34.1 & 234 & 243 & & \\
\hline 6 & -23.7 & 400 & 383 & & \\
\hline 7 & -17.4 & 633 & & & \\
\hline 8 & -13.3 & 939 & & & \\
\hline 9 & -10.5 & 1359 & & & \\
\hline 10 & -8.52 & 1845 & & & \\
\hline \multirow[t]{4}{*}{$\tau_{1}^{*}$} & & 1.85 & & & \\
\hline & \multirow{2}{*}{\multicolumn{5}{|c|}{$\begin{array}{c}\mathrm{Au}(111) \\
E_{\text {up }}=-1.95 \mathrm{eV}, V_{g}=4.20 \mathrm{eV}\end{array}$}} \\
\hline & & & & & \\
\hline & \multicolumn{2}{|c|}{ This study } & WPP & \multicolumn{2}{|c|}{ Experiment } \\
\hline$n$ & $E_{n}$ & $\tau_{n}$ & $\tau_{n}$ & $E_{n}$ & $\tau_{n}$ \\
\hline \multirow[t]{2}{*}{1} & -808 & 1.59 & 1.60 (Ref. 35) & $-800 \pm 30($ Ref. 41$)$ & 3 (Ref. 41) \\
\hline & & & 1.62 (Ref. 36) & $-800($ Ref. 36$)$ & $4 \pm 1($ Ref. 30$)$ \\
\hline \multirow[t]{2}{*}{2} & -209 & 10.3 & 10.5 (Ref. 35) & & \\
\hline & & & 10.8 (Ref. 36) & & \\
\hline 3 & -93.8 & 33.3 & 33.7 (Ref. 35) & & \\
\hline 4 & -53.0 & 78.5 & 79.9 (Ref. 35) & & \\
\hline 5 & -33.9 & 154 & 168 (Ref. 35) & & \\
\hline 6 & -23.6 & 263 & 298 (Ref. 35) & & \\
\hline 7 & -17.3 & 417 & & & \\
\hline 8 & -13.3 & 620 & & & \\
\hline 9 & -10.5 & 899 & & & \\
\hline 10 & -8.50 & 1217 & & & \\
\hline$\tau_{1}^{*}$ & & 1.22 & & & \\
\hline
\end{tabular}

The calculated energies and lifetimes of IPRs on $\mathrm{Ag}(111)$ are shown in Table I. By construction of the pseudopotential, the energy of the $n=1$ IPS ( $\left.E_{n=1}=-756 \mathrm{meV}\right)$ coincides with experimental value. ${ }^{39}$ The calculated binding energies of the $n=2,3, \ldots, 7$ IPRs are also in excellent agreement with experiments. We obtain the lifetimes of the $n=2,3,4$ IPRs in reasonable agreement with photoemission data, however, for the $n \geqslant 5$ IPRs, the calculated lifetimes are shorter than the measured ones. For $\mathrm{Cu}(111)$, early TR-2PPE experiments ${ }^{6,8}$ were able to resolve only the $n=2$ and 3 IPRs (Table I), and good agreement is observed between those experiments and present calculations both for binding energies and lifetimes. 
TABLE II. The energies $E_{n}(\mathrm{meV})$ and lifetimes $\tau_{n}$ (fs) of IPRs. Present results are compared with wave-packet propagation (WPP) calculations and with experimental data. $E_{\text {up }}$ is the position of the upper edge of the projected band gap, $V_{g}$ is the width of the projected band gap.

\begin{tabular}{|c|c|c|c|c|c|c|c|c|c|c|c|c|c|c|c|}
\hline \multirow[t]{3}{*}{$\begin{array}{l}E_{\mathrm{up}}(\mathrm{eV}) \\
V_{g}(\mathrm{eV}) \\
\end{array}$} & \multicolumn{4}{|c|}{$\begin{array}{c}\mathrm{Al}(001) \\
-5.65 \\
1.68 \\
\end{array}$} & \multicolumn{2}{|c|}{$\begin{array}{c}\mathrm{Al}(111) \\
-8.54 \\
0.30\end{array}$} & \multicolumn{3}{|c|}{$\begin{array}{c}\mathrm{Be}(0001) \\
-3.95 \\
6.20\end{array}$} & \multicolumn{2}{|c|}{$\begin{array}{c}\operatorname{Mg}(0001) \\
-4.66 \\
0.70\end{array}$} & \multicolumn{2}{|c|}{$\begin{array}{c}\mathrm{Na}(110) \\
-1.55 \\
0.67\end{array}$} & \multicolumn{2}{|c|}{$\begin{array}{c}\operatorname{Li}(110) \\
-0.11 \\
2.99\end{array}$} \\
\hline & \multicolumn{2}{|c|}{ This study } & \multicolumn{2}{|c|}{ Experiment (Ref. 40) } & \multicolumn{2}{|c|}{ This study } & \multicolumn{2}{|c|}{ This study } & \multirow{2}{*}{$\begin{array}{c}\text { WPP (Ref. 35) } \\
\tau_{n}\end{array}$} & \multicolumn{6}{|c|}{ This study } \\
\hline & $E_{n}$ & $\tau_{n}$ & $E_{n}$ & $\tau_{n}$ & $E_{n}$ & $\tau_{n}$ & $E_{n}$ & $\tau_{n}$ & & $E_{n}$ & $\tau_{n}$ & $E_{n}$ & $\tau_{n}$ & $E_{n}$ & $\tau_{n}$ \\
\hline 1 & Not vis & sible & Not vi & isible & Not vi & sible & -988 & 0.87 & 0.73 & Not $v$ & isible & -795 & 1.00 & -733 & \\
\hline 2 & -210 & 4.73 & $-210 \pm 10$ & $9 \pm 6 \mathrm{fs}$ & -213 & 3.43 & -235 & 6.45 & 6.09 & -213 & 4.09 & -219 & 5.15 & -220 & \\
\hline 3 & -94.3 & 15.5 & $-91 \pm 6$ & & -95.6 & 12.8 & -101 & 22.0 & 18.1 & -94.9 & 13.3 & -97.5 & 16.4 & -99.8 & 115 \\
\hline 4 & -53.5 & 37.2 & $-57 \pm 8$ & $35 \pm 15$ fs & -54.1 & 30.7 & -56.4 & 53.6 & 53.5 & -53.8 & 31.8 & -54.9 & 39.1 & -55.4 & 207 \\
\hline 5 & -34.3 & 74.7 & $-36 \pm 9$ & & -34.6 & 62.5 & -35.7 & 107 & 110 & -34.4 & 64.4 & -34.9 & 78.2 & -35.2 & 374 \\
\hline 6 & -23.8 & 125 & & & -24.0 & 103 & -24.6 & 184 & 191 & -23.9 & 106.5 & -24.2 & 131 & -24.3 & 624 \\
\hline 7 & -17.5 & 201 & & & -17.6 & 170 & -18.0 & 295 & & -17.5 & 172 & -17.7 & 210 & -17.8 & 972 \\
\hline 8 & -13.4 & 300 & & & -13.4 & 247 & -13.7 & 440 & & -13.4 & 255 & -13.5 & 312 & -13.6 & 1434 \\
\hline 9 & -10.6 & 426 & & & -10.6 & 349 & -10.8 & 642 & & -10.6 & 360 & -10.7 & 447 & -10.7 & 2056 \\
\hline 10 & -8.55 & 586 & & & -8.60 & 486 & -8.73 & 889 & & -8.58 & 500 & -8.64 & 616 & -8.65 & 2789 \\
\hline$\tau_{1}^{*}$ & & 0.58 & & & & 0.48 & & 0.86 & & & 0.52 & & 0.61 & & 2.91 \\
\hline
\end{tabular}

On the $\mathrm{Au}(111), \mathrm{Al}(001), \mathrm{Al}(111), \mathrm{Be}(0001), \mathrm{Mg}(0001)$, and $\mathrm{Na}(110)$ surfaces, the entire series of IPSs lies in resonance with projected bulk states so that the IPRs are formed. The lifetimes of the $n=1$ resonances appear very short due to the low reflectivity of the metal-vacuum interface. The corresponding resonance peaks are too broad and barely distinguishable from the nonresonant background. In some cases explicitly mentioned in the Table II, extraction of their energies and lifetimes is impossible. As an example, let us consider the Green's function of $\mathrm{Al}(001)$. The left panel of Fig. 2 shows $G_{\mathrm{IP}}(\omega)$ in the energy range between -1500 and $-500 \mathrm{meV}$. Obviously, the pole structure is not visible so that the properties of the $n=1$ IPR can not be extracted. The $n=2$ resonance appears as a weak shoulder in $\operatorname{Im}\left[G_{\mathrm{IP}}(\omega)\right]$ linked with projected density of states (PDOS). Exploiting both the real and imaginary parts of the Green's function, we could however characterize the corresponding pole at $\omega_{n=2}=-210-70 i(\mathrm{meV})$.
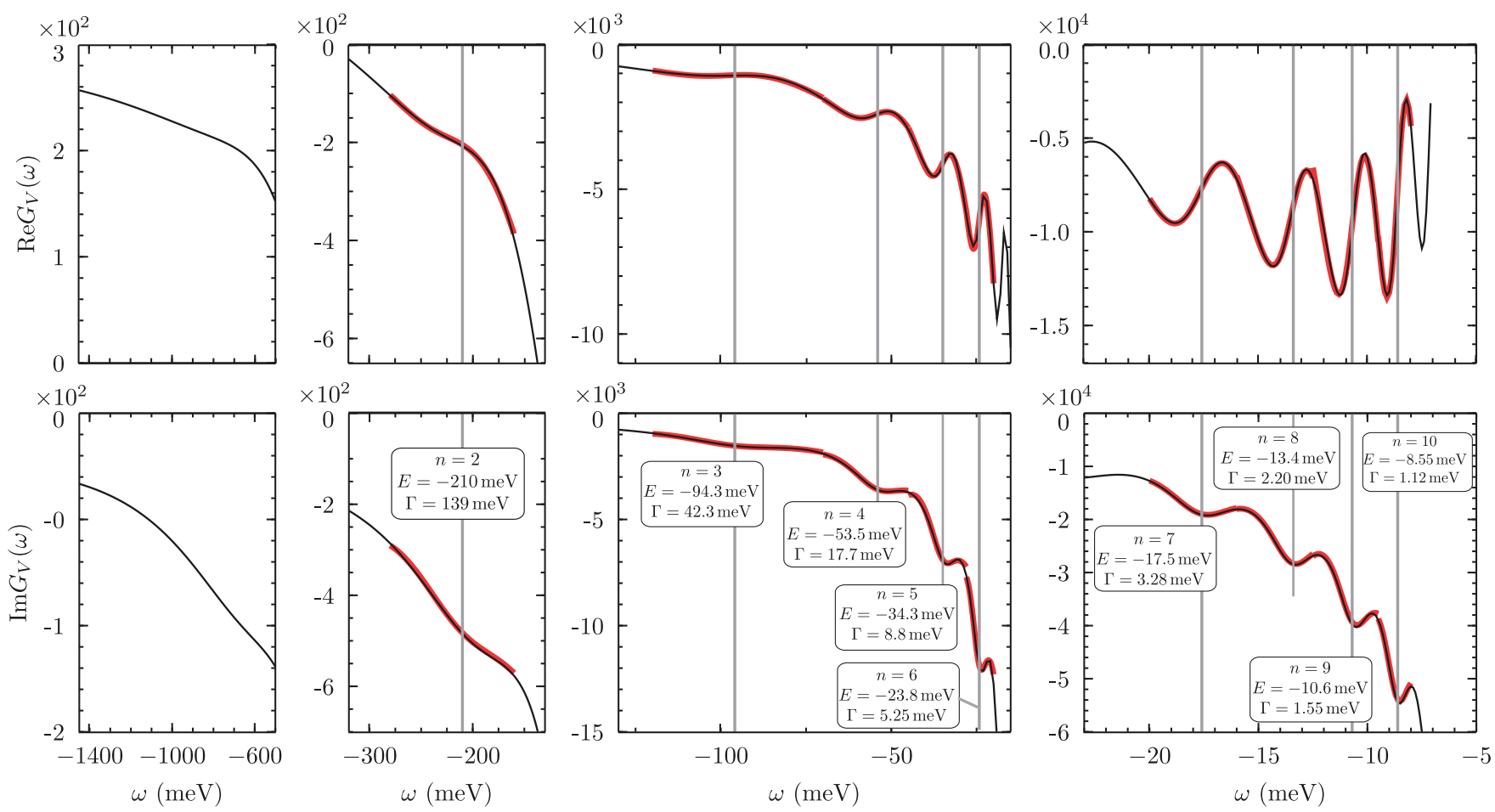

FIG. 2. (Color online) The real (upper panel) and imaginary (lower panel) parts of $G_{\mathrm{IP}}(\omega)$ for $\mathrm{Al}(001)$. Black thin lines: the calculated dependence; thick red lines: the fits by function (24) in the vicinities of the resonances. 
The longest lifetime $\tau_{n=1}=1.59$ fs of the first IPR is observed on the $\mathrm{Au}(111)$ surface. Earlier photoemission estimations ${ }^{30,41}$ based on the linewidth of the resonance predicted longer lifetimes $\tau \geqslant 3$. This discrepancy may be attributed to experimental difficulties, connected with measurements of such short-lived states. On $\operatorname{Li}(110)$ the upper edge of the projected bulk gap is located just $0.11 \mathrm{eV}$ below the vacuum lavel, and the $n=1$ and 2 IPSs reside in the gap. The $n \geqslant 3$ IPRs are then very close to the gap edge, where reflectivity of the metal-vacuum interface is high. These states possess then the longest lifetimes on $\operatorname{Li}(110)$ as compared to all other surfaces studied in this work. The above trend is also seen in the $\operatorname{Ag}(111), \mathrm{Cu}(111)$, and $\mathrm{Au}(111)$ data shown in Table I: the closer is the top of the projected band gap to the vacuum level, the longer are the lifetimes over the entire series of the IPRs.

While for the surfaces of aluminium and magnesium we can not identify the position of the $n=1$ IPR, higher resonances $(n \geqslant 3)$ are more pronounced allowing extraction of their properties. Corresponding energies and lifetimes are given in Table II. Recently, Winter et al. ${ }^{40}$ performed time-resolved two-photon photoemission study of the IPRs on $\mathrm{Al}(001)$. They also did not find any sign of the $n=1$ IPR, while the $n=2,3,4,5$ IPRs have been observed, and the measured energies obey Eq. (1) with the quantum defect $\delta=0$. The lifetimes of IPRs reported in Ref. 40 were obtained with two distinct procedures:

(i) The lifetimes were estimated by fitting the experimental data to a model, containing as a free parameter the coupling strength of IPRs to the bulk continuum. Thus, the $\tau_{n}=$ $0.8 \mathrm{fs} \times n^{3}$ law was obtained. These lifetimes are slightly longer than the values obtained in this study where we find the $\tau_{n}=0.58 \mathrm{fs} \times n^{3}$ law.

(ii) The lifetimes of the $n=2$ and 4 IPRs were also derived in Ref. 40 from the rate-equation model. The resulting values are given in Table II. These lifetimes are also longer than our results, although our calculated lifetime of the $n=2$ IPR agrees with the experimental one within the error bar.

It is worth to mention that on the $\mathrm{Al}(111), \mathrm{Al}(001)$, and $\operatorname{Mg}(0001)$ surfaces the linewidths of the IPRs are larger than their energy separation. Thus, the theoretical and experimental extractions of the lifetimes of the IPRs at these surfaces may lack accuracy.

The calculated lifetimes of the image potential resonances are presented in Fig. 3 on a logarithmic scale. It is clearly seen that for large quantum numbers $n$ the lifetimes scale as $\tau_{n}=\tau_{1}^{*} \times n^{3}$. Deviations from this law are observed for IPRs with small quantum numbers. The values $\tau_{1}^{*}$ obtained by fitting the calculated data for $n \geqslant 4$ are given in Tables I and II. Because of the aforementioned deviation of the low- $n$ lifetimes from the power law, $\tau_{1}^{*} \neq \tau_{n=1}$, where $\tau_{n=1}$ is the calculated lifetime of the $n=1$ IPR. Note the tendency for an overall increase of the lifetimes ( $\tau_{1}^{*}$ is larger) for surfaces with broader projected band gap located closer to the vacuum level. In accord with this observation, the shortest lifetimes are observed on jelliumlike surfaces, such as $\mathrm{Al}(001), \mathrm{Al}(111)$, and $\operatorname{Mg}(0001)$, where the projected band gap is very small and resides several $\mathrm{eV}$ below the vacuum level.

For $\mathrm{Ag}(111), \mathrm{Cu}(111), \mathrm{Au}(111)$, and $\mathrm{Be}(0001)$ surfaces, we can compare results obtained from the present GF analysis with
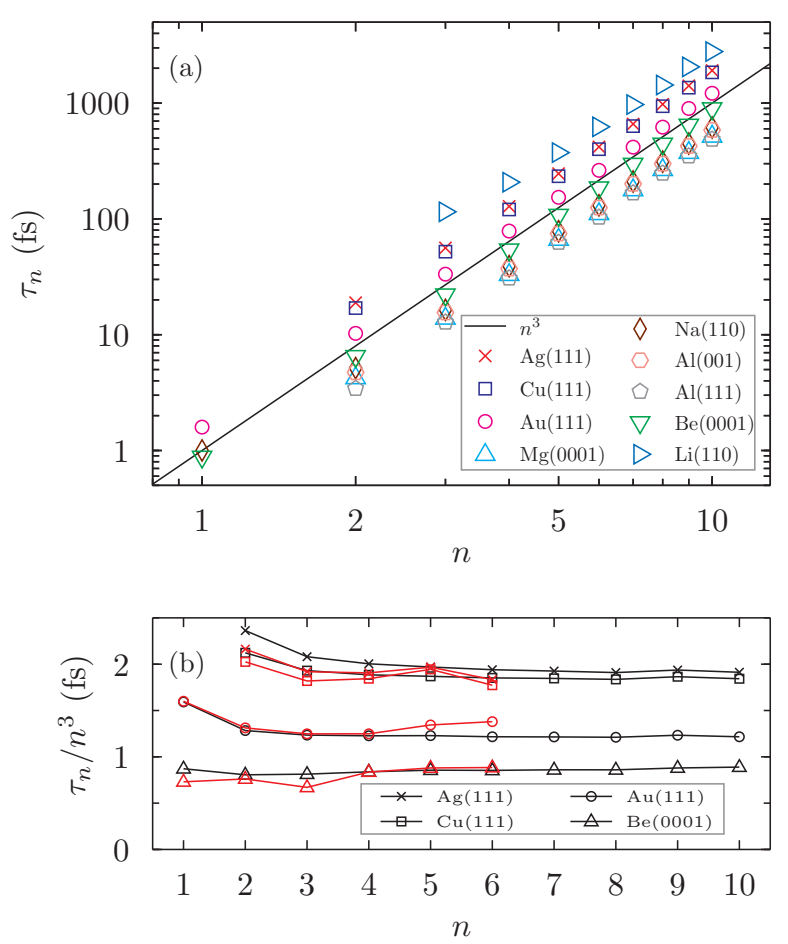

FIG. 3. (Color online) (a) The calculated lifetimes of image potential resonances on close-packed surfaces. The line $\tau=1 \mathrm{fs} \times n^{3}$ is a guide to the eye. (b) $\tau_{n} / n^{3}$ obtained in this work (black lines) and in Ref. 35 (red lines).

WPP studies. ${ }^{35,36}$ The energies of the states agree better than to within $1 \%$. As to the one-electron lifetimes, for $\operatorname{Ag}(111)$, $\mathrm{Cu}(111)$, and $\mathrm{Au}(111)$ our results agree with WPP results within $10 \%$ overall. This is a satisfactory accuracy provided different treatment of the nonresonant contribution and a different way to extract the widths of the resonances. Note that for $\operatorname{Ag}(111)$ we use slightly modified parameters of the pseudopotential as compared to those given in Ref. 51, and used in WPP. ${ }^{35}$ We checked that with the $\operatorname{Ag}(111)$ potential parametrization as given in Ref. 51 and as used in the WPP study, we retrieve the WPP results. We obtain $\tau_{2}=17.6 \mathrm{fs}$, $\tau_{3}=52.9 \mathrm{fs}, \tau_{4}=121 \mathrm{fs}, \tau_{5}=233 \mathrm{fs}$, and $\tau_{6}=396 \mathrm{fs}$. For $\mathrm{Be}(0001)$ with the same potential ${ }^{51,58}$ as Borisov et al..$^{35}$ we obtain the lifetimes of the first and the third IPRs which are $\approx 20 \%$ longer. We tentatively attribute this discrepancy to the inevitable errors in extraction of the decay rates of the short-lived quasistationary states. Note, that the decay rate $\hbar / \tau_{n=1}=756 \mathrm{meV}$ of the $n=1$ image potential resonance on $\mathrm{Be}(0001)$ is comparable to the energy difference of the first two resonances $E_{n=2}-E_{n=1}=753 \mathrm{meV}$. The calculated resonant structures strongly overlap, rendering difficult the extraction of the poles of the GF. We believe that the numerical accuracy of the present calculation is higher than in Ref. 35 . Indeed, we use both real and imaginary parts of the image potential GF for the pole extraction, while the WPP procedure relies on the analysis of the projected density of states. The latter strongly depends on the proper choice of the initial state for the time propagation. ${ }^{35}$ The accuracy claim is confirmed by data treatment shown in Fig. 3(b). The $\tau_{n} / n^{3}$ quantity obtained in this work is a smooth monotonous 

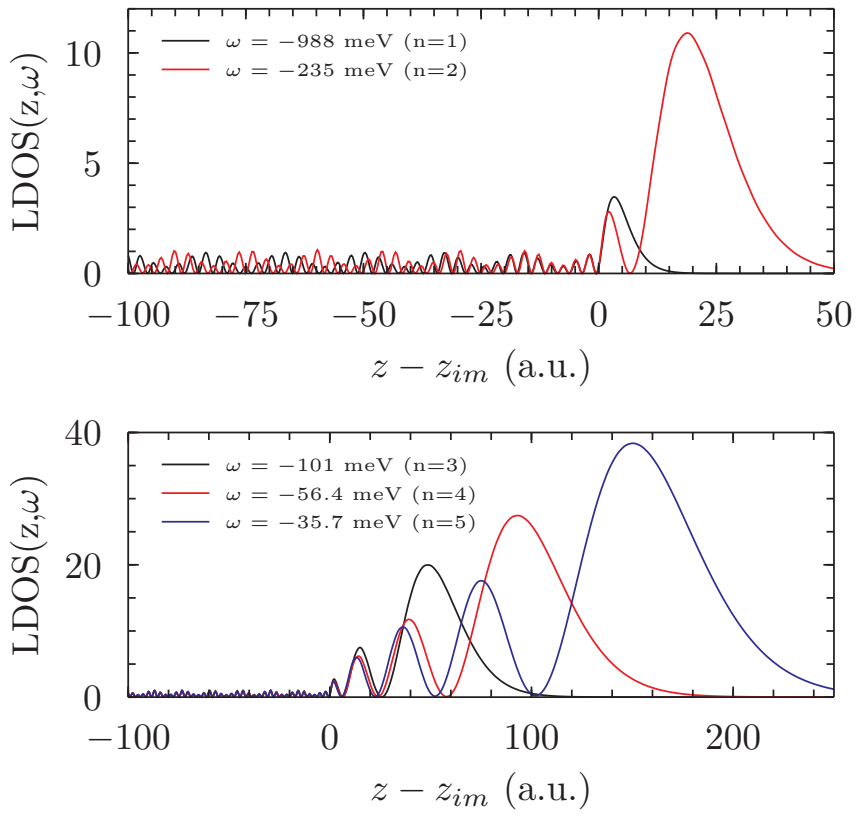

FIG. 4. (Color online) Local density of states $\operatorname{LDOS}(z, \omega)=$ $-\operatorname{Im}[G(z, z, \omega)] / \pi$ at the resonance energies on $\operatorname{Be}(0001)$.

function of $n$ converging towards $\tau_{1}^{*}$, while the WPP values exhibit unsystematic oscillations reflecting numerical inaccuracies.

Figure 4 shows the local $z$-dependent density of states $\operatorname{LDOS}(z, \omega)=-\operatorname{Im}[G(z, z, \omega)] / \pi$ of $\operatorname{Be}(0001)$, calculated at the energies $E_{n}$, corresponding to the IPRs. For $z>z_{i m}$, the $\operatorname{LDOS}\left(z, E_{n}\right)$ basically corresponds to the electron density distributions of the IPSs with the same quantum numbers $n$ on the surfaces, where all IPSs reside within the energy gap, e.g., $\mathrm{Cu}(100)$ or $\mathrm{Ag}(100)$. Indeed, the IPRs can be seen as the IPSs bound by the image potential above the metal and coupled to the bulk continuum because of the finite reflectivity of the metal-vacuum interface. As an example, one can compare Fig. 4 with the probability distribution of the IPSs on $\mathrm{Cu}(100) .{ }^{51,58}$ At $z<z_{\text {im }}$, the local density of states is formed by the bulk electronic states with the corresponding energy and reflects the continuum contribution to the resonance wave function. As the quantum number $n$ grows, additional nodal structures appear in the vacuum part of the electron density distribution, the wave function is shifted into the vacuum, and the density amplitude of the continuum part decreases as $\propto n^{-3}$. The latter reflects the $n^{-1.5}$ dependence of the discrete state (IPS) coupling with metal continuum and the $n^{-3}$ dependence of the resonance decay rates. ${ }^{40}$

This result allows us to give an estimate for the many-body contribution to the decay of the IPRs. Indeed, we have observed that the vacuum part of the resonance wave functions is basically that of the corresponding image potential state, and that the residue of the pole in $G_{\mathrm{IP}}(\omega)$ is nearly unity, reflecting only small contribution of the continuum part to the resonance state. Thus, as zero-order approximation, we can consider the wave function of the IPR above the surface as the unity normalized wave function of the bound IPS. The many-body theory developed for the IPSs (Ref. 26) then applies leading to $\tau_{1}^{*} \simeq 30$ fs for the inelastic decay

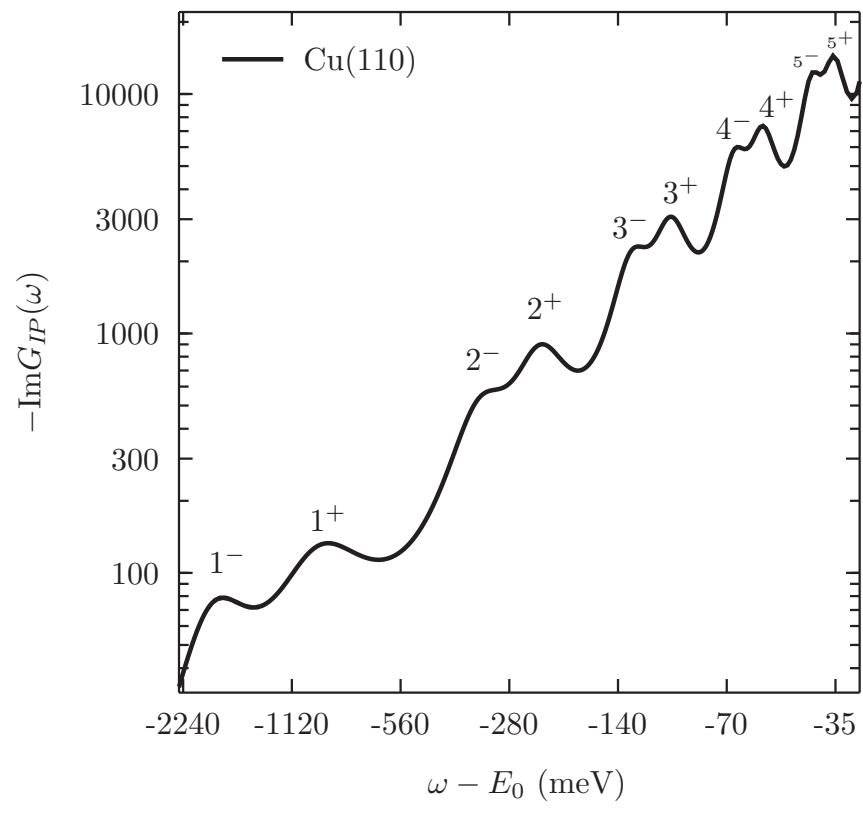

FIG. 5. Imaginary part of the image potential GF $\mathcal{N}(\omega)=$ $-\operatorname{Im}\left[G_{\mathrm{IP}}(\omega)\right]$ for $\mathrm{Cu}(110)$ surface. $E_{0}=(\pi / a)^{2} / 2$ is the kinetic energy of free electrons at the $\bar{Y}$ point. Double logarithmic scale is used.

due to electron-electron scattering, as can be extracted from the experimental and theoretical data on $\mathrm{Cu}(100), \operatorname{Ag}(100)$, and $\mathrm{Au}(100)$ surfaces. ${ }^{11,26,67,68}$ Due to the similarity of the resonance wave function with that of IPS, we expect that the inelastic lifetimes of IPRs are the same order of magnitude, i.e., $\tau_{n}^{e-e} \sim 30 \mathrm{fs} \times n^{3}$. The rates of one-electron resonant decay into the metal, as obtained here, are larger by an order of magnitude than the many-body decay rate estimated above. Thus, these are one-electron resonant transitions that determine the lifetimes of the IPRs for the surfaces studied in this work. As to the electron-phonon scattering, it results in a very small contribution to the decay rate of the conventional IPSs. For example, $\Gamma_{e-p h} \lesssim 1 \mathrm{meV}$ (Refs. 33 and 48 ) has been reported for the first IPS. Hence, we expect that $\Gamma_{e-p h}$ is also negligible for the IPRs.

TABLE III. The calculated energies $E_{n}$ and lifetimes $\tau_{n}$ of the IPRs at the $\overline{\mathrm{Y}}$ point on $\mathrm{Cu}(110) . E_{\text {up }}$ and $V_{g}$ stand for, respectively, the energy of the upper edge and for the width of the projected band gap. $E_{0}=\frac{1}{2}\left(\frac{\pi}{a}\right)^{2}$ is the energy of free electrons at the $\overline{\mathrm{Y}}$ point.

\begin{tabular}{lcccc}
\hline \hline \multicolumn{5}{c}{$\mathrm{Cu}(110)$} \\
$E_{0}=2877 \mathrm{meV}$ \\
\\
\hline$n$ & $E_{n-}(\mathrm{meV})$ & $\tau_{n-}(\mathrm{fs})$ & $E_{n+}(\mathrm{meV})$ & $\tau_{n}+(\mathrm{fs})$ \\
\hline 1 & 1162 & 0.62 & 1944 & 1.20 \\
2 & 2552 & 4.8 & 2648 & 7.5 \\
3 & 2752 & 18 & 2779 & 25 \\
4 & 2812 & 47 & 2823 & 60 \\
5 & 2838 & 104 & 2843 & 118 \\
$\tau_{1}^{*}$ & & 0.71 & & 0.94 \\
\hline \hline
\end{tabular}




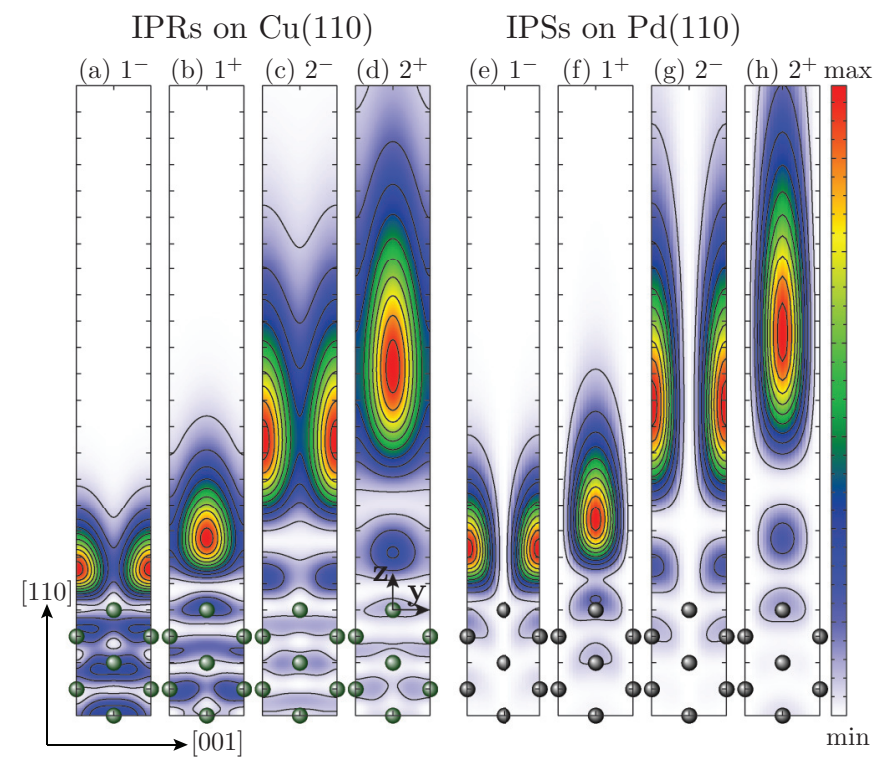

FIG. 6. (Color online) (a)-(d) Local density of states $\operatorname{LDOS}(y, z, \omega)=-\operatorname{Im} G\left(y, z, y, z ; k_{\bar{Y}}, \omega\right) / \pi$ at the resonance energies at the $\overline{\mathrm{Y}}$ point on $\mathrm{Cu}(110)$. (e)-(h) Charge density distribution of IPSs on $\operatorname{Pd}(110)$ (Ref. 63). Color scale is renormalized for each subfigure independently.

\section{B. $\mathrm{Cu}(110)$ surface}

On the $\mathrm{Cu}(110)$ surface, the energy gap in the projection of the bulk band structure on the SBZ at the $\bar{Y}$ point is located below the vacuum level. On the other hand, the IPSs at the $\bar{Y}$ point are above the vacuum level because kinetic energy of the free-electron motion parallel to the surface equals to $E_{0}=\frac{1}{2}\left(\frac{\pi}{a}\right)^{2}=2.877 \mathrm{eV}$ at the $\overline{\mathrm{Y}}$ point. Thus, IPRs are formed above the vacuum level. We stress here that while the total energy of the electrons in IPRs is positive (above the vacuum level), the energy associated with motion perpendicular to the surface remains negative, so that the electrons are bound by the image potential.

Figure 5 shows the imaginary part of the image potential GF linked with probability to find an electron in the image potential region $\mathcal{N}(\omega)=-\operatorname{Im}\left[G_{\mathrm{IP}}(\omega)\right]$ for energies $\omega$ below $E_{0}$. The function $\mathcal{N}(\omega)$ exhibits peaks, corresponding to the IPRs. Analogous to Ref. 57, we denote these IPRs as $1^{-}, 1^{+}$, $2^{-}, 2^{+}, \ldots$ in the energy ascending order. Following, we will explain the meaning of such notation. One can see that the energy difference of the $n^{-}$and $n^{+}$resonances is significantly smaller than the energy separation between the $n^{ \pm}$and $m^{ \pm}$ resonances characterized by quantum numbers $m \neq n$.

The energies and lifetimes of IPRs on $\mathrm{Cu}(110)$ are determined in a procedure analogous to that on close-packed surfaces. To extract the positions of the two close-situated poles $\omega_{n+}$ and $\omega_{n-}$ more accurately the two-pole fitting function was used:

$$
\tilde{G}_{\mathrm{IP}}(\omega)=\frac{1}{\omega-\omega_{n-}}+\frac{1}{\omega-\omega_{n+}}+\sum_{l=0}^{2} A_{l}\left(\omega-\omega_{n}\right)^{l} .
$$

The resulting energies and lifetimes of the IPRs are given in Table III.

Figures 6(a)-6(d) show the $\operatorname{LDOS} \operatorname{LDOS}(y, z, \omega)=$ $-\operatorname{Im} G(y, z, y, z, \omega) / \pi$ of $\mathrm{Cu}(110)$, calculated at energies $\omega=$ $E_{n \pm}$ corresponding to the IPRs. The $\mathrm{Cu}(110)$ result is qualitatively similar to the charge density distributions of IPSs obtained at the $\bar{Y}$ point on Pd(110) (Ref. 63) shown in Figs. 4(e)-4(h). At the $\bar{Y}$ point, the IPSs and IPRs are of either even $\left(n^{+}\right)$or odd $\left(n^{-}\right)$symmetry with respect to the $y=0$ mirror plane. The charge density of odd states is zero at $y=0$ and reaches maximum at $y=a / 2$. Reversely, the charge density of even states is maximum at $y=0$ and zero at $y=a / 2$. The dependence of the charge density on the $z$ coordinate perpendicular to the surface ([110] direction) is similar for the $n^{+}$and $n^{-}$states with the same $n$. As for the IPRs at the $\bar{\Gamma}$ point, increasing $n$ leads to an additional node appearing in the $z$ dependence of the electronic densities on the vacuum side, and the continuum part inside the metal quickly weakens. Observe that the $n^{+}$states have smaller binding energy for the motion perpendicular to the surface $E_{0}-E_{n+}$ and thus extend further into the vacuum. Precisely, this larger extension into the vacuum leads to stronger decoupling from the metal as compared to the $n^{-}$states. As a consequence,
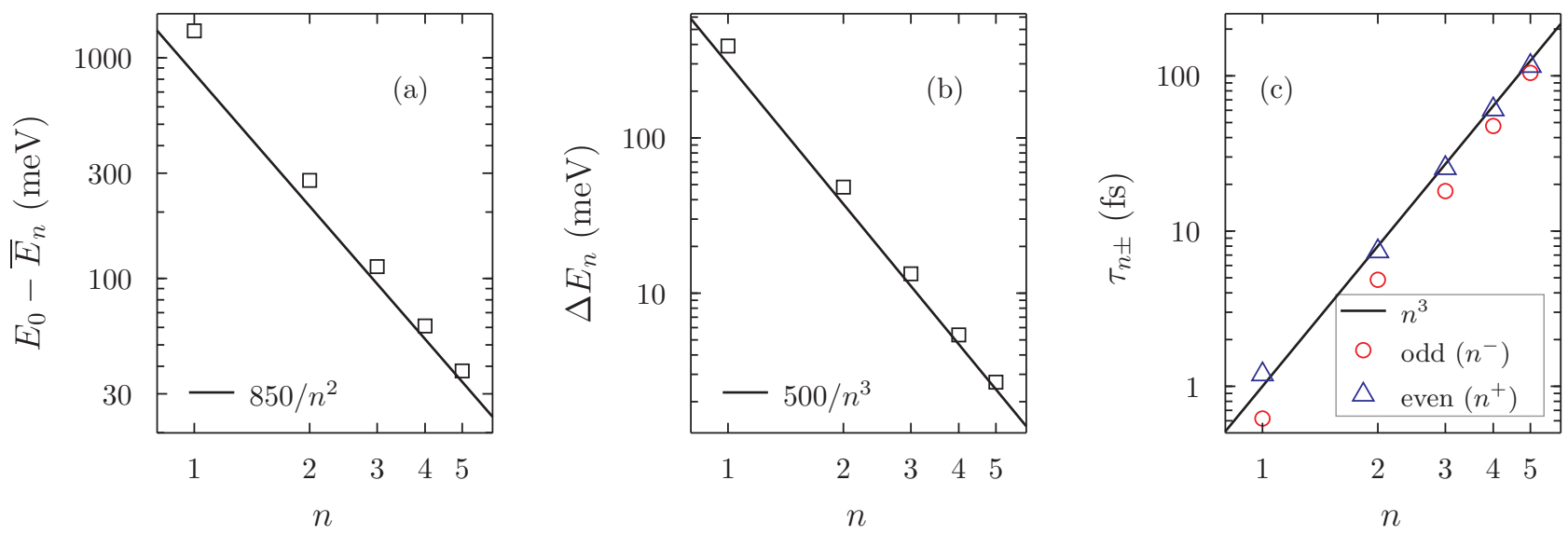

FIG. 7. (Color online) (a) Average energies $\bar{E}_{n}=\left(E_{n+}+E_{n-}\right) / 2$; (b) energy differences $\Delta E_{n}=E_{n+}-E_{n-}$; (c) lifetimes of the IPRs at the $\bar{Y}$ point. Results are shown as function of the principal quantum number $n$. In panel (a) the energies are given relatively to the kinetic energy of free electrons at the $\bar{Y}$ point $E_{0}=\frac{1}{2}\left(\frac{\pi}{a}\right)^{2}$. Double logarithmic scale is used. 
the $n^{+}$IPRs on $\mathrm{Cu}(110)$ possess longer lifetimes against one electron decay into the metal.

Figure 7 shows on a logarithmic scale the $n$ dependence of the average energies of the IPR pairs $\bar{E}_{n}=\left(E_{n+}+E_{n-}\right) / 2$, of the energy differences $\Delta E_{n}=E_{n+}-E_{n-}$, and of the lifetimes $\tau_{n}^{ \pm}$of the IPRs. The average energy of the IPR follows the Rydberg series converging to $E_{0}$ as $E_{0}-\bar{E}_{n} \propto n^{-2}$. For $n \geqslant 2$, the energies $\bar{E}_{n}$ are well described by Eq. (1) upon replacement $E_{\mathrm{vac}} \rightarrow E_{0}$, and with quantum defect $\delta=-0.25$. The energy splitting of the even and odd states with the same $n$ evolves as $\Delta E_{n} \propto n^{-3}$. This is because the splitting of the states originates from the interaction with the $y$-dependent potential inside the crystal and in the surface region. At the same time, the electrons in high- $n$ IPRs reside mainly in vacuum and the attempt rate to probe the surface region drops as $n^{-3}$ (Ref. 35). The decoupling from the metal also results in conventional $\tau_{n \pm}=\tau_{1 \pm}^{*} \times n^{3}$ quantum number $n$ dependence of the lifetimes of IPRs at the $\bar{Y}$ point as shown in Fig. 3(c). This is analogous to IPRs at the $\bar{\Gamma}$ on the close-packed surfaces. The values $\tau_{1 \pm}^{*}$ differ by a factor of 1.3 for even and odd IPRs (see Table III). This difference is due to the different localization of odd and even IPRs as discussed above.

\section{SUMMARY}

We presented the study of the image potential resonances at metal surfaces. With the Green's function technique, we obtained the binding energies $E_{n}$ of these quasistationary states and their one-electron decay rates via resonant electron transfer into the bulk. We performed extensive calculations of $E_{n}$ and lifetimes $\tau_{n}$ of IPRs at the $\bar{\Gamma}$ point on a number of closepacked metal surfaces, and at the $\bar{Y}$ point on the $\mathrm{Cu}(110)$ surface. The method developed here outperforms the wavepacket propagation technique applied earlier and allows us to confidently address the IPRs with high quantum numbers $n$. In this study, results with $n$ up to 10 are reported.
We show that at the $\overline{\mathrm{Y}}$ point on $\mathrm{Cu}(110)$ the IPRs with the same quantum number $n$ are split, forming even and odd states. The energy difference between even and odd states is proportional to $n^{-3}$. The average energy of a pair of resonances with $n \geqslant 2$ can be well described by the $\bar{E}_{n}=\left(E_{n+}+E_{n-}\right)=$ $E_{0}-0.85 \mathrm{eV} /(n+\delta)^{2}$ Rydberg sequence, where $\delta=-0.25$ is the quantum defect, and $E_{0}$ is the energy of the electron motion parallel to the surface at the $\bar{Y}$ point. The spatial profile of the electron density of IPRs on $\mathrm{Cu}(110)$ is found similar to that of IPSs on $\operatorname{Pd}(110)$.

The calculated lifetimes demonstrate the scaling law $t_{n} \propto$ $n^{3}$ both for close-packed and $\mathrm{Cu}(110)$ surfaces. Our values are in agreement with the experimental data, as well as with the results obtained with the wave-packet propagation method (when available). By comparing results obtained for different metal surfaces, we confirm the general trend: the closer is the IPRs series to the edge of the projected band gap, the higher is the reflectivity of the metal-vacuum interface and the longer are the resonance lifetimes through the entire series.

We have shown that the vacuum part of the electron densities of the IPRs, particularly of the long-lived resonances with high quantum numbers $n$, is basically the same as for the IPSs located in the bulk projected energy gap. The continuum contribution to the resonance wave function is very small and decays as $n^{-3}$. Therefore, many-body decay rates of the IPRs can be estimated from the experimental and calculated data for conventional IPSs. We thus conclude that the resonant one-electron transfer into the bulk is the leading channel for the population decay of the IPRs on studied surfaces.

\section{ACKNOWLEDGMENTS}

We acknowledge financial support of the Ministry of Education and Science of the Russian Federation (state Task No. 2.8575.2013), the University of the Basque Country UPV/EHU (Grant No. GIC07-IT-366-07), the Departamento de Educación del Gobierno Vasco, and the Spanish Ministerio de Ciencia e Innovación (Grant No. FIS2010-19609-C02-01).
*Corresponding author: tsirkinss@gmail.com

${ }^{1}$ Laser Spectroscopy and Photochemistry on Metal Surfaces, edited by H. L. Dai and W. Ho (World Scientific, Singapore, 1995).

${ }^{2}$ H. Nienhaus, Surf. Sci. Rep. 45, 1 (2002).

${ }^{3}$ M. Bonn, S. Funk, Ch. Hess, D. N. Denzler, C. Stampfl, M. Scheffler, M. Wolf, and G. Ertl, Science 285, 1042 (1999).

${ }^{4}$ E. V. Chulkov, V. M. Silkin, and M. Machado, Surf. Sci. 482-485, 693 (2001).

${ }^{5}$ L. Vitali, P. Wahl, M. A. Schneider, K. Kern, V. M. Silkin, E. V. Chulkov, and P. M. Echenique, Surf. Sci. 523, L47 (2003).

${ }^{6}$ M. Wolf, E. Knoesel, and T. Hertel, Phys. Rev. B 54, R5295 (1996). ${ }^{7}$ Th. Fauster, Surf. Sci. 507-510, 256 (2002).

${ }^{8}$ M. Weinelt, J. Phys.: Condens. Matter 14, R1099 (2002).

${ }^{9}$ P. Szymanski, S. Garrett-Roe, and C. Harris, Prog. Surf. Sci. 78, 1 (2005).

${ }^{10}$ T. Fauster, M. Weinelt, and U. Höfer, Prog. Surf. Sci. 82, 224 (2007).
${ }^{11}$ U. Höfer, I. L. Shumay, Ch. Reuß, U. Thomann, W. Wallauer, and Th. Fauster, Science 277, 1480 (1997).

${ }^{12}$ S. Link, H. A. Dürr, G. Bihlmayer, S. Blügel, W. Eberhardt, E. V. Chulkov, V. M. Silkin, and P. M. Echenique, Phys. Rev. B 63, 115420 (2001).

${ }^{13}$ A. Schäfer, I. L. Shumay, M. Wiets, M. Weinelt, Th. Fauster, E. V. Chulkov, V. M. Silkin, and P. M. Echenique, Phys. Rev. B 61, 13159 (2000).

${ }^{14}$ P. de Andrés, P. M. Echenique, and F. Flores, Phys. Rev. B 35, 4529 (1987).

${ }^{15}$ J. D. McNeill, R. L. Lingle, N.-H. Ge, C. M. Wong, R. E. Jordan, and C. B. Harris, Phys. Rev. Lett. 79, 4645 (1997).

${ }^{16}$ E. V. Chulkov, J. Kliewer, R. Berndt, V. M. Silkin, B. Hellsing, S. Crampin, and P. M. Echenique, Phys. Rev. B 68, 195422 (2003).

${ }^{17}$ C. Corriol, V. M. Silkin, D. Sánchez-Portal, A. Arnau, E. V. Chulkov, P. M. Echenique, T. von Hofe, J. Kliewer, J. Kröger, and R. Berndt, Phys. Rev. Lett. 95, 176802 (2005). 
${ }^{18}$ P. S. Kirchmann, L. Rettig, X. Zubizarreta, V. M. Silkin, E. V. Chulkov, and U. Bovensiepen, Nat. Phys. 6, 782 (2010).

${ }^{19}$ S. Mathias, A. Ruffing, F. Deicke, M. Wiesenmayer, I. Sakar, G. Bihlmayer, E. V. Chulkov, Y. M. Koroteev, P. M. Echenique, M. Bauer, and M. Aeschlimann, Phys. Rev. Lett. 104, 066802 (2010).

${ }^{20}$ H. Petek, M. J. Weida, H. Nagano, and S. Ogawa, Science 288, 1402 (2000).

${ }^{21}$ M. Bauer, S. Pawlik, and M. Aeschlimann, Phys. Rev. B 60, 5016 (1999).

${ }^{22}$ C. Frischkorn and M. Wolf, Chem. Rev. 106, 4207 (2006).

${ }^{23}$ J. Gauyacq, A. Borisov, and M. Bauer, Prog. Surf. Sci. 82, 244 (2007).

${ }^{24}$ R. Díez Muiño, D. Sánchez-Portal, V. M. Silkin, E. V. Chulkov, and P. M. Echenique, Proc. Natl. Acad. Sci. USA 108, 971 (2011).

${ }^{25}$ S. Achilli, M. I. Trioni, E. V. Chulkov, P. M. Echenique, V. Sametoglu, N. Pontius, A. Winkelmann, A. Kubo, J. Zhao, and H. Petek, Phys. Rev. B 80, 245419 (2009).

${ }^{26}$ E. V. Chulkov, A. G. Borisov, J. P. Gauyacq, D. Sánchez-Portal, V. M. Silkin, V. P. Zhukov, and P. M. Echenique, Chem. Rev. 106, 4160 (2006).

${ }^{27}$ M. W. Finnis, R. Kaschner, C. Kruse, J. Furthmuller, and M. Scheffler, J. Phys.: Condens. Matter 7, 2001 (1995).

${ }^{28}$ I. D. White, R. W. Godby, M. M. Rieger, and R. J. Needs, Phys. Rev. Lett. 80, 4265 (1998).

${ }^{29}$ P. M. Echenique and J. B. Pendry, J. Phys. C: Solid State Phys. 11, 2065 (1978).

${ }^{30}$ T. Fauster and W. Steinmann, in Electromagnetic Waves: Recent Development in Research, edited by P. Halevi (Elsevier, Amsterdam, 1995), p. 350.

${ }^{31}$ P. M. Echenique, R. Berndt, E. V. Chulkov, Th. Fauster, A. Goldmann, and U. Höfer, Surf. Sci. Rep. 52, 219 (2004).

${ }^{32}$ I. Yu. Sklyadneva, R. Heid, K.-P. Bohnen, and E. V. Chulkov, New J. Phys. 11, 103038 (2009).

${ }^{33}$ I. Yu. Sklyadneva, S. S. Tsirkin, S. V. Eremeev, R. Heid, K.-P. Bohnen, and E. V. Chulkov, Phys. Solid State 53, 2508 (2011).

${ }^{34}$ S. Heers, P. Mavropoulos, S. Lounis, R. Zeller, and S. Blügel, Phys. Rev. B 86, 125444 (2012).

${ }^{35}$ A. G. Borisov, E. V. Chulkov, and P. M. Echenique, Phys. Rev. B 73, 073402 (2006).

${ }^{36}$ C. Lindstrom, D. Quinn, and X.-Y. Zhu, J. Chem. Phys. 122, 124714 (2005).

${ }^{37}$ D. C. Marinica, C. Ramseyer, A. G. Borisov, D. Teillet-Billy, J. P. Gauyacq, W. Berthold, P. Feulner, and U. Höfer, Phys. Rev. Lett. 89, 046802 (2002).

${ }^{38}$ F. E. Olsson, A. G. Borisov, M. Persson, N. Lorente, A. K. Kazansky, and J. P. Gauyacq, Phys. Rev. B 70, 205417 (2004).

${ }^{39}$ M. Marks, C. H. Schwalb, K. Schubert, J. Güdde, and U. Höfer, Phys. Rev. B 84, 245402 (2011).

${ }^{40}$ M. Winter, E. V. Chulkov, and U. Höfer, Phys. Rev. Lett. 107, 236801 (2011).
${ }^{41}$ Ch. Reuß, W. Wallauer, and Th. Fauster, Surf. Rev. Lett. 3, 1547 (1996).

${ }^{42}$ C. Harris, N.-H. Ge, R. Lingle, Jr., J. McNeill, and C. Wong, Annu. Rev. Phys. Chem. 48, 711 (1997).

${ }^{43}$ L. Jurczyszyn, Surf. Sci. 247, 158 (1991)

${ }^{44}$ L. Jurczyszyn, Surf. Sci. 259, 65 (1991).

${ }^{45}$ L. Jurczyszyn and M. Stęślicka, Surf. Sci. 376, L424 (1997).

${ }^{46}$ A. Damm, K. Schubert, J. Güdde, and U. Höfer, Phys. Rev. B 80, 205425 (2009).

${ }^{47}$ K. Duncker, M. Kiel, and W. Widdra, Surf. Sci. 606, L87 (2012).

${ }^{48}$ S. S. Tsirkin, S. V. Eremeev, E. V. Chulkov, M. Marks, K. Schubert, J. Güdde, and U. Höfer, Phys. Rev. B 86, 085424 (2012).

${ }^{49}$ W. Berthold, U. Höfer, P. Feulner, E. V. Chulkov, V. M. Silkin, and P. M. Echenique, Phys. Rev. Lett. 88, 056805 (2002).

${ }^{50}$ K. Schubert, A. Damm, S. V. Eremeev, M. Marks, M. Shibuta, W. Berthold, J. Güdde, A. G. Borisov, S. S. Tsirkin, E. V. Chulkov, and U. Höfer, Phys. Rev. B 85, 205431 (2012).

${ }^{51}$ E. V. Chulkov, V. M. Silkin, and P. M. Echenique, Surf. Sci. 437, 330 (1999).

${ }^{52}$ A. Goldmann, M. Donath, W. Altmann, and V. Dose, Phys. Rev. B 32, 837 (1985).

${ }^{53}$ R. A. Bartynski and T. Gustafsson, Phys. Rev. B 33, 6588 (1986).

${ }^{54}$ W. Jacob, V. Dose, U. Kolac, Th. Fauster, and A. Goldmann, Z. Phys. B 63, 459 (1986).

${ }^{55}$ N. V. Smith, C. T. Chen, J. M. Tranquada, and P. D. Johnson, Phys. Rev. B 38, 12259 (1988).

${ }^{56}$ C. T. Chen and N. V. Smith, Phys. Rev. B 35, 5407 (1987).

${ }^{57}$ N. V. Smith, C. T. Chen, and M. Weinert, Phys. Rev. B 40, 7565 (1989).

${ }^{58}$ E. V. Chulkov, V. M. Silkin, and P. M. Echenique, Surf. Sci. 391, L1217 (1997).

${ }^{59}$ J. Kliewer, R. Berndt, E. V. Chulkov, V. M. Silkin, P. M. Echenique, and S. Crampin, Science 288, 1399 (2000).

${ }^{60}$ I. A. Nechaev, M. F. Jensen, E. D. L. Rienks, V. M. Silkin, P. M. Echenique, E. V. Chulkov, and Ph. Hofmann, Phys. Rev. B 80, 113402 (2009).

${ }^{61}$ E. V. Chulkov, I. Sarría, V. M. Silkin, J. M. Pitarke, and P. M. Echenique, Phys. Rev. Lett. 80, 4947 (1998).

${ }^{62}$ S. S. Tsirkin, S. V. Eremeev, and E. V. Chulkov, Phys. Solid State 52, 188 (2010).

${ }^{63}$ S. S. Tsirkin, S. V. Eremeev, and E. V. Chulkov, Surf. Sci. 604, 804 (2010).

${ }^{64}$ S. S. Tsirkin, S. V. Eremeev, and E. V. Chulkov, Phys. Rev. B 84, 115451 (2011)

${ }^{65}$ S. Tsirkin, S. Eremeev, and E. Chulkov, J. Exp. Theor. Phys. 115, 673 (2012).

${ }^{66}$ J. Inglesfield, J. Phys. C: Solid State Phys. 4, L14 (1971).

${ }^{67}$ I. L. Shumay, U. Höfer, C. Reuß, U. Thomann, W. Wallauer, and T. Fauster, Phys. Rev. B 58, 13974 (1998).

${ }^{68}$ K. Boger, M. Weinelt, and T. Fauster, Phys. Rev. Lett. 92, 126803 (2004). 